stomatologie $2013 \cdot 110: 7-7$

DOI 10.1007/s00715-012-0194-9

๑) Springer-Verlag Wien 2013

\author{
H. Haider \\ Österreichische Gesellschaft für Zahn-, Mund- und Kieferheilkunde, Markt Allhau
}

\title{
Die neue stomatologie
}

\section{The new stomatologie}

Sehr geehrte Frau Kollegin, sehr geehrter Herr Kollege!

Eines unserer drei Versprechen bei der letzten Wahl des Vorstandes des Dachverbandes der ÖGZMK war, die Zeitschrift stomatologie „praxisfreundlicher“ zu gestalten.

Nach vielen Vorgesprächen, Vorbereitungen, Anregungen und Überarbeitungen halten Sie nun die erste Nummer der neuen stomatologie in Ihren Händen. Sie ist der Versuch, ein 110 Jahre altes zahnärztliches Journal so neu zu präsentieren, dass es für den Zahnarzt praxistaugliche Information auf gesicherter wissenschaftlicher Basis vermittelt. Die einzelnen Hefte werden immer einen Themenschwerpunkt haben.

\section{》) Wissenschaft und Praxis sind untrennbar miteinander verbunden}

Sowohl durch qualitativ hochstehende Originalarbeiten als auch durch kurze Abstracts in der Rubrik „Für Sie gelesen“ wollen wir die neue stomatologie für die Kollegenschaft attraktiver machen. Völlig neu ist die Möglichkeit, „Fortbildungspunkte“ für das „zahnärztliche Fortbildungsdiplom“ zu erwerben: Fortbildung bequem zu Hause, ohne zeitliche Einschränkung und ohne zusätzliche Kosten. Ebenso neu ist die Rubrik „Wie löse ich diesen Fall?“. Dazu werden Kliniker und Niedergelassene ihre Diagnosen und Therapievorschläge zur Diskussion stellen. Auch Sie selbst sind eingeladen, „Ihren persönlichen Kommentar“ zu diesen Fällen oder anderen Themen be- kannt zu geben. Ich hoffe, dass die neue stomatologie so Ihre Akzeptanz findet.

Ebenso bewusst ist uns aber auch die Tatsache, dass die ÖGZMK ein „wissenschaftlicher" Verein ist. Wissenschaft und Praxis sind so untrennbar miteinander verbunden wie die beiden Seiten einer Medaille.

Um auch dem wissenschaftlichen Anspruch gerecht zu werden, werden wir die bisherigen wissenschaftlichen Beiräte durch nationale und internationale Erweiterung einerseits, aber auch durch zahlenmäßige Straffung und fachspezifische Ausrichtung andererseits neu strukturieren.

Die Führung eines Vereines und dessen wissenschaftliche Betreuung erfordern ein grundsätzlich anderes Anforderungsprofil. Um erfolgreich für die ÖGZMK im Interesse ihrer Mitglieder arbeiten zu können, ist eine gute Kooperation zwischen Vereinsleitung und wissenschaftlichem Beirat unerlässlich.

Die neue stomatologie als wissenschaftliches Journal kann nur dann die Akzeptanz der Mitglieder finden, wenn die Leser das Gefühl haben, praxisnahe Information auf hohem Niveau zu erhalten.

Der Erstversuch ist gestartet. Für Wünsche, Anregungen und Beschwerden sind wir dankbar. Denn die neue stomatologie soll auch als Basis für die Kommunikation zwischen Wissenschaft und Praxis, zwischen dem ÖGZMK-Vorstand und den Teilorganisationen, aber ganz besonders zwischen allen Mitgliedern der ÖGZMK dienen.

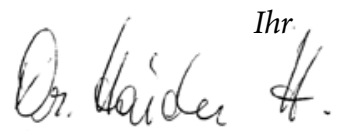

Prof. Dr. Herbert Haider

Präsident

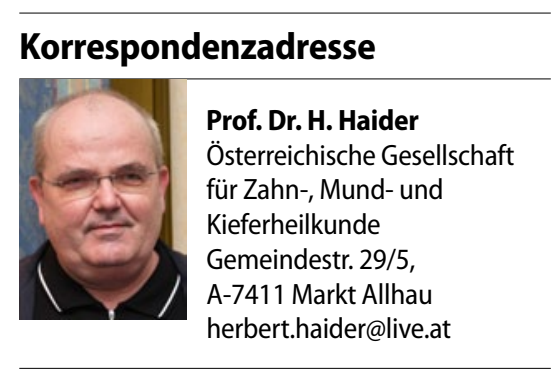

